\title{
Gestión de la información territorial municipal a través del catastro multifinalitario
}

\author{
Diego Erba \\ Universidad Nacional del Litoral (Santa Fe-Argentina) \\ diegoerba@gmail.com
}

Fecha de presentación: 2 de febrero de 2015 • Fecha de aceptación: 3 de noviembre de 2015

Artículo de investigación 


\section{Diego Erba}

\section{Resumen}

El primer catastro territorial fue implementado en América Latina en 1824. A partir de entonces, y durante el resto del siglo XIX, 8 países crearon instituciones públicas con funciones relativas a la publicidad inmobiliaria, mientras que los otros 11 países restantes comenzaron a trabajar su información territorial a partir del siglo XX. En la mayoría de los países de la región los sistemas catastrales nacieron estructurados y continúan desarrollándose, bajo el modelo básico que solo contempla datos económicos, valor del suelo y de las mejoras; físicos, forma, dimensiones y ubicación de los inmuebles; y jurídicos, propietarios y poseedores.

Palabras clave: catastro, publicidad inmobiliaria, información territorial.

JEL: J11 Tendencias y previsiones demográficas; R11 Actividad económica regional: crecimiento, desarrollo y cambios; R31 Oferta y mercados de la vivienda.

\section{Asbtract}

The first territorial land was implemented in Latin America in 1824. Since then, and during the rest of the nineteenth century, 8 countries created public institutions with functions related to real estate advertising, while the other 11 countries began to work their land information only from the twentieth century. In most countries of the region cadastral systems they were born structured (and continue to develop) under the basic model that only includes economic data, land value and improvements; physical, shape, dimensions and location of the property; and legal, owners and holders.

Keywords: cadastral, real estate advertising, territorial information.

JEL: J11 Demographic trends and forecasts; R11 regional economic activity: growth, development and change; R31 Supply and housing markets.

\section{Resumo}

A primeira terra territorial foi implementada na América Latina em 1824. Desde então, e durante o resto do século XIX, 8 países criaram instituições públicas com as funções relacionadas com a publicidade imobiliário, enquanto os outros 11 países começou a trabalhar suas informações terras apenas a partir do século XX. Na maioria dos países os sistemas região cadastrais eles nasceram estruturados (e continuar a desenvolver), sob o modelo básico que inclui apenas os dados econômicos, o valor da terra e melhorias; físicas, forma, dimensões e localização da propriedade; e legais, proprietários e titulares.

Palavras-chave: cadastral, publicidade imobiliária, informação territorial.

JEL: J11 As tendências demográficas e previsões; R11 actividade económica regional: crescimento, desenvolvimento e mudança; R31 Mercados de abastecimento e de habitação. 


\section{Introducción}

$\mathrm{E}$ 1 término catastro aparece en varios textos constitucionales de la región. En el Ecuador está presente en los art. 264 y 375 y Disposiciones Transitorias, lo cual evidencia su relevancia para la administración pública; también se verifica que un alto porcentaje de las leyes catastrales mencionan el término multifinalitario o su filosofía asociada. Además, es muy frecuente que, en los congresos y seminarios internacionales sobre catastro, la multifinalidad forme parte de las principales sesiones. El modelo de catastro territorial multifinalitario (CTM), surgido en Europa después de la II Guerra Mundial, aterrizó en Latinoamérica al final del siglo pasado y se esparce con velocidad creciente, aunque de forma despareja en la región. El modelo amplió el número de variables territoriales registradas y actualmente diversas jurisdicciones lo adoptaron, inspiradas por la eficiencia de este instrumento, en la definición de políticas públicas orientadas a mejorar el planeamiento y el financiamiento urbano con inversiones mínimas. En Ecuador el término "multifinalitario" se usa desde los años de 1970, aunque el modelo no se ha implementado aún en su integridad. El contexto actual es inmejorable para la estructuración de un sistema nacional de catastro basado en el modelo CTM, pues se percibe una creciente madurez conceptual de funcionarios y técnicos, además de buena voluntad política. Manteniendo las potestades municipales, cada administración puede desarrollar su forma de implementarlo, incorporando aliados y sumando aspectos del catastro territorial de forma paulatina y modular, siempre dentro de los parámetros establecidos por el ente rector: el Ministerio de Desarrollo Urbano y Vivienda (MIDUVI).

La integración de datos del modelo CTM trae numerosos beneficios y construye el camino más corto para que los administradores conozcan los aspectos económicos, físicos, jurídicos, ambientales y sociales de su jurisdicción. La realidad modelada por el CTM correlaciona la información te- 
rritorial, propicia un mejor planeamiento urbano, amplía las alternativas de financiamiento urbano, agiliza el mercado inmobiliario, y optimiza el uso de recursos tecnológicos y humanos. Los catastros territoriales requieren más de una modernización filosófica y conceptual que de una modernización tecnológica; requieren más de articulación institucional que de recursos financieros. El primer desafío para la implementación del modelo CTM es priorizar el salto administrativo y procedimental, lo cual no necesariamente implica un cambio de legislación. Las reformas estructurales en América Latina han sido condicionadas a la promulgación de reglamentos, de nuevas leyes o a alteraciones de la legislación catastral vigente. Esa visión legalista acabó atrasando las acciones. Un desafío es avanzar de forma más pragmática y con mayor celeridad a partir, por ejemplo, de la difusión de directrices de adopción optativa a fin de crear "cultura CTM" de forma paulatina. En este sentido, es importante destacar que gran parte de las soluciones ya se encuentran en la legislación vigente, es cuestión de identificarlas y luego priorizar los desafíos de implementación. La instrumentación del modelo CTM implica cambios de paradigmas en la gestión económica, tecnológica, administrativa y profesional de cada jurisdicción. El orden y la cantidad de desafíos depende de cada realidad, pero la experiencia muestra que los cuatro desafíos más urgentes que los administradores de los catastros ecuatorianos deberían encarar son: elaborar mapas de valor referidos al mercado inmobiliario; incorporar nuevas geo-tecnologías bajo una visión jurídica y biescalar para la cartografía; formar profesionales en las diferentes facetas del CTM; y administrar el territorio de forma coordinada. En Ecuador, el Decreto Ejecutivo 688, publicado en el Registro Oficial, Suplemento (ROS), No. 410, de 22 de marzo de 2011 establece, en el art. 1, la creación del Sistema Nacional de Catastro Integrado Geo Referenciado de Hábitat y Vivienda, con el objetivo de registrar de forma sistemática, lógica, georreferenciada y ordenada, en una base de datos integral e integrada, los catastros urbanos y rurales, que sirva como herramienta para la formulación de políticas de desarrollo urbano. La misma norma, en su art. 2, establece que la rectoría del Sistema Nacional de Catastro será ejercida por el MIDUVI, el cual queda facultado para expedir políticas nacionales y los actos administrativos para el correcto funcionamiento del Sistema en el marco de la Constitución, la ley y el decreto ejecutivo, el cual coordinará con los gobiernos autónomos descentralizados. En este sentido el MIDUVI se encuentra trabajando junto 
a los principales actores del quehacer catastral del Ecuador con el objetivo de redactar las directrices que permitan implementar el Sistema Nacional de Catastro, el cual será basado en el modelo multifinalitario.

\section{Modelos catastrales}

América Latina es una región donde el uso del suelo presenta marcados contrastes: la extensa selva del Amazonas frente a sus crecientes áreas de deforestación, grandes regiones despobladas frente a densas concentraciones urbanas, la coexistencia de la riqueza y de la pobreza en los mismos vecindarios, entre otros. Esta situación deriva, por una parte, del proceso de colonización caracterizado por la explotación y la ocupación de tierras a cualquier precio, y por otra, del desarrollo de políticas de suelos establecidas por intereses parciales (o aún la falta de políticas), proceso que ha sido facilitado por la persistencia de registros de tierras desactualizados y/o distorsionados. Esa desinformación territorial impacta, aunque con mayor sutileza que la corrupción, en las ciudades latinoamericanas las cuales se ven afectadas por la falta de seguridad en la tenencia de la tierra y por la desigualdad que existe en el cobro de tributos. El panorama no es hegemónico en la región $\mathrm{y}$, si bien aún son pocos los sistemas latinoamericanos que generan información territorial completa para las múltiples necesidades del Estado y de los particulares, es perceptible el incremento de responsabilidad que se le está dando a los catastros territoriales como instituciones proveedoras de datos referidos al predio, que es la célula básica del territorio. Si bien los primeros catastros territoriales fueron organizados con fines meramente recaudatorios, numerosos cambios se han producido en las visiones institucionales y, consecuentemente, en el rol de los catastros dentro de la administración pública. La concepción del catastro como depósito de planos para salvaguarda de derechos sobre la tierra o como la masa de datos que conforma la base de la fiscalidad inmobiliaria está cambiando, paulatinamente, hacia la visión multifinalitaria. Actualmente, aunque con diferentes niveles de intensidad, existen movimientos que procuran transformar los catastros territoriales básicos en sistemas de información modernos y multifinalitarios, desvinculándolos de conceptos fiscalistas para llevarlos hacia un concepto universal de bases de datos territoriales disponibles para todos. Estos catastros pasan 
a representar una herramienta holística de planificación que puede usarse a nivel local, regional y nacional con la finalidad de abordar asuntos relevantes como la expansión urbana, la disminución de la pobreza, las políticas de suelo y el desarrollo comunitario sostenible.

\section{El modelo catastral básico}

Las bases de datos que a nivel de predio corresponden a tres aspectos que conforman el modelo básico económico-físico-jurídico heredado de Europa aún caracteriza gran parte de los catastros latinoamericanos y se encuentra ampliamente difundido en Ecuador. El objetivo de este modelo ha sido estructurar datos económicos, físicos y jurídicos de los predios, a partir de los cuales se desarrollen políticas públicas destinadas a ordenar el territorio (figura 1).

Figura 1

\section{Definición de la política tributaria Aspectos del catastro territorial básico y procesos asociados}

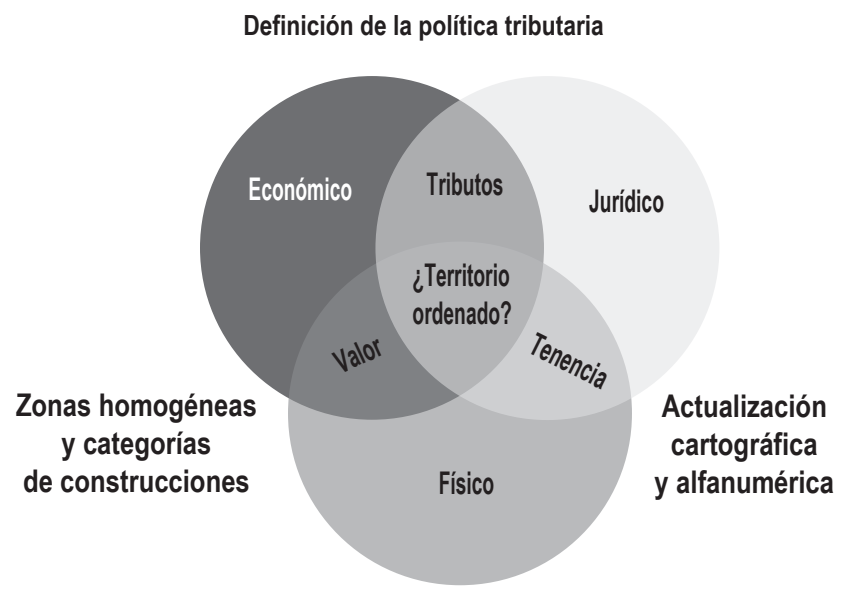

Fuente: el autor.

Elaboración propia. 
El catastro económico -también conocido erróneamente como catastro fiscal- registra, en sus bases, datos que permiten determinar el valor de los inmuebles a través de diferentes métodos y, a partir de ellos, definir la política de financiamiento urbano. Entre las variables que influyen en la conformación del valor de un inmueble se pueden señalar su localización, la forma y dimensiones del terreno, y las características y tamaño de las construcciones como elementos esenciales. En los aspectos económicos del catastro, la mayoría de las jurisdicciones continúan trabajando bajo el paradigma de valuar con fines fiscales a través del método de reposición el mismo que se encuentra mencionado en el Código Orgánico de Organización Territorial, Autonomía y Descentralización (COOTAD, art. 502), manteniendo en bancos de datos separados el valor del suelo y de las construcciones. Gran parte del atraso y de la inequidad que muestran los valores en relación con los practicados en el mercado inmobiliario se debe justamente a esta decisión administrativa, puesto que el método de reposición exige complejas bases de datos, difíciles de mantener actualizadas. En los últimos años se ha diseminado el uso de la econometría asociada a la geoestadística para el desarrollo de valuaciones masivas, principalmente en Brasil y Colombia, con los cuales se construyen los mapas de valores. Estos mapas necesitan de una base cartográfica que puede ser construida a partir de relevamientos topográficos, geodésicos, fotogramétricos o satelitales.

El catastro físico, también denominado catastro geométrico por algunos autores, está conformado por documentos cartográficos y bases alfanuméricas que caracterizan al terreno y las construcciones de cada predio. En el aspecto físico del catastro se evidencia una rápida popularización del uso de equipamientos de levantamiento digital. Estaciones totales y receptores GPS han transformado la actividad de los profesionales habilitados, quienes pasan a medir tiempos en vez de ángulos y distancias, obteniendo directamente coordenadas que pueden ser transformadas a cualquier sistema de referencia y/o proyección cartográfica, simplificando enormemente la representación de los predios. La popularización de la fotogrametría digital, los levantamientos con láser escáner aerotransportados y el uso de imágenes satelitales de alta resolución están contribuyendo a la actualización.

Con el pasar del tiempo, los administradores percibieron que el catastro tenía una función mucho más relevante aún que registrar datos físicos y 
económicos, y pasaron a organizarlo como complemento de los Registros de la Propiedad. Surgió así el catastro jurídico, esta denominación ha llevado a considerar que el catastro territorial debe registrar también los títulos de propiedad, lo cual llevaría a duplicaciones sin sentido. Además, es importante destacar que el catastro trabaja sobre el objeto de derecho y no sobre el sujeto ni sobre el derecho en sí. Napoleón fue uno de los precursores de este nuevo aspecto del catastro y manifestó su interés de colocarlo al servicio del Derecho Civil en su Código, diciendo: "un buen catastro parcelario será el complemento del Código en lo que se refiere a la posesión del suelo. Es necesario que los mapas sean suficientemente precisos y detallados para fijar límites y evitar litigios" (Francia 1804).

Desde el punto de vista catastral existen dos límites: el límite de la posesión efectiva, el cual es determinado por el uso del predio y es materializado por entes naturales (ríos, mar, entre otros) o antropológicos (paredes, cercas, entre otros); y el límite jurídico, que corresponde a una línea imaginaria que no puede ubicarse en el terreno sin una señal que la materialice. En este sentido el dimensionamiento de un predio consiste en la definición geométrica de ambos límites, siendo esta una condición imprescindible para poder integrarlo dentro del rompecabezas del territorio representado en la cartografía catastral. Un predio se considera físicamente dimensionado y precisamente ubicado cuando se conocen sus medidas lineares y angulares, y su posicionamiento, el cual puede ser relativo (no georreferenciado) o absoluto (georreferenciado). Paralelamente, para determinar el límite legal es necesario realizar el estudio de los títulos del predio en cuestión y de los títulos vecinos. La figura 2 muestra la interpretación gráfica del posicionamiento relativo de un predio, en la cual la realidad de campo -denominada en algunos países estado de hecho- no coincide con la realidad jurídica descripta en el título de propiedad ("estado de derecho").

En la figura 2 se evidencian tres polígonos cuando en realidad debería ser uno. Esta confusión de límites es bastante generalizada en el Ecuador y revertirla exige un cambio en la estructura y administración de los catastros territoriales, comenzando por la adopción de dos escalas de representación, una predial, más general, usada para planeamiento; y otra de detalle, apropiada para los fines físico-jurídicos.

En este contexto, la falta de relación entre los valores catastrales y los practicados en el mercado inmobiliario, y la incertidumbre sobre la tenencia 
del suelo derivada de la desconexión y falta de correlación de datos entre los registros de la propiedad y los catastros, son pruebas contundentes de que este modelo básico ha sido insuficiente para definir políticas de suelo. Fueron estas y otras carencias que llevaron a desarrollar un modelo catastral más amplio, que contempla datos provenientes de múltiples fuentes y productores, los cuales pueden ser correlacionados para generar información a múltiples usuarios.

Figura 2

Posicionamiento relativo de un predio Confusión de límites causados por las situaciones de hecho y de derecho

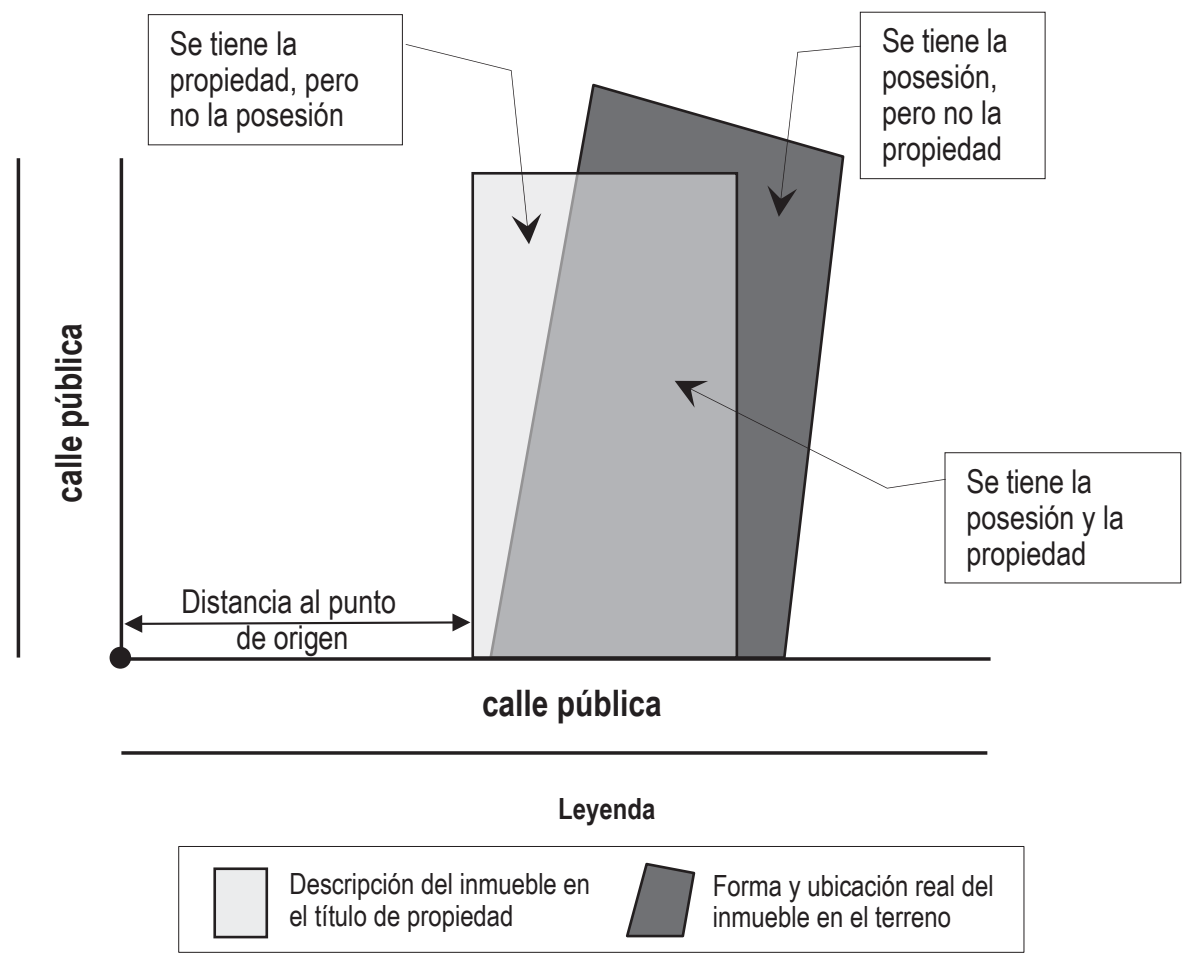

Fuente: el autor.

Elaboración propia. 


\section{El modelo multifinalitario}

Los catastros territoriales del mundo han experimentado diferentes procesos de reestructuración y actualización durante las últimas décadas. Las leyes nacionales y locales de catastro en América Latina han incorporado términos como multifinalidad, Infraestructura de Datos Espaciales (IDE) y georreferenciación pero, en la práctica, su implementación es lenta. Las administraciones suman aspectos multitemáticos en diferentes ritmos, lo cual es perfectamente válido puesto que el catastro multifinalitario es modular, no excluyente y no tiene una forma única de ser estructurado. Esa es una de las principales bondades del modelo CTM que se estructura como complemento del catastro básico.

La multifinalidad se construye interconectando el catastro básico económico-físico-jurídico a los aspectos estructurales que constan en las bases de datos de los catastros temáticos. Estos catastros, también denominados sectoriales, puesto que son estructurados y mantenidos por diferentes sectores de la administración pública o empresas privadas, igualmente enfocan sus

Figura 3

\section{Aspectos del Catastro Territorial Multifinalitario}

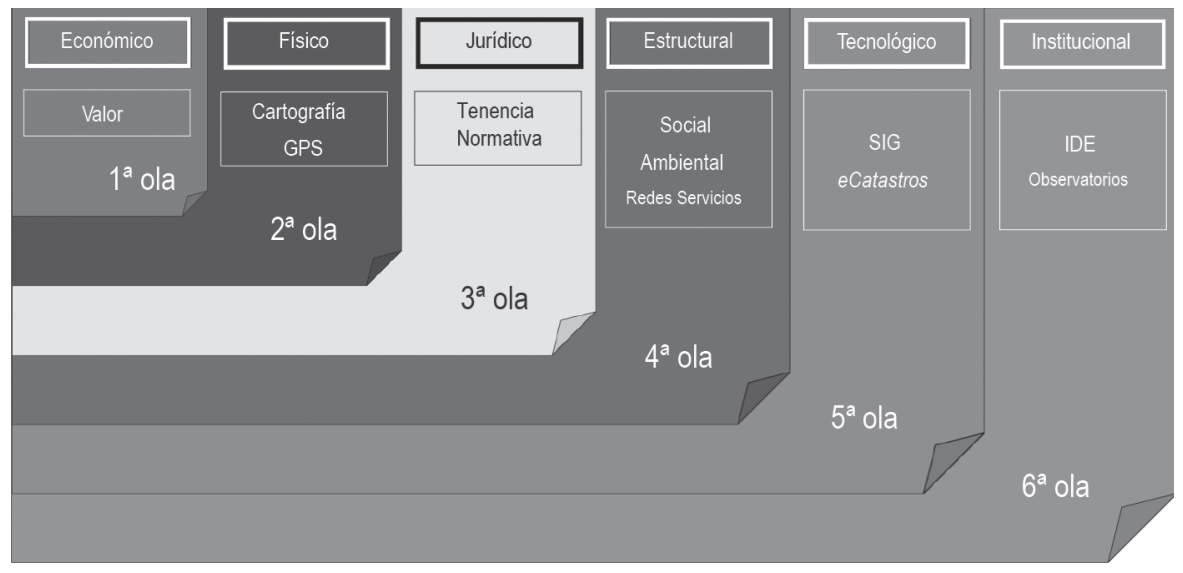

Fuente: el autor.

Elaboración propia. 
bases de datos en el predio, aunque generalmente desconectados del catastro municipal (figura 3).

Entre los aspectos estructurales se encuentran los ambientales, las redes de servicios y viales, y el perfil social de los ocupantes de los predios. El catastro ambiental normalmente está compuesto por datos que permiten identificar las características y los recursos naturales existentes en cada predio. En algunas jurisdicciones el aspecto catastral está compuesto por catastros temáticos como el catastro minero, muy desarrollado en Perú, los catastros de tipos suelos, depósitos geológicos, elementos hidrográficos, espacios forestales, entre otros. En algunas ciudades es posible encontrar los catastros de áreas verdes e inclusive de árboles históricos. Frecuentemente los recursos naturales son tratados como bienes gratuitos, pues se consideran propiedad de todos. La ausencia de los derechos de propiedad bien establecidos sobre los recursos imposibilita la asignación adecuada de un valor. No obstante, los servicios que esos recursos prestan sí pueden ser valuados. Los servicios de los ecosistemas son definidos como aquellos beneficios que recibe el ser humano por la utilización de diferentes elementos de la naturaleza, los cuales pueden estar comprendidos en ecosistemas silvestres o antrópicos, y cuyos efectos en la calidad de vida son tangibles e intangibles (Gómez y De Groot 2007). Muchos de estos servicios son transados directamente en el mercado de inmuebles mientras que otros, a pesar de reconocerse como vitales para la operatividad del ecosistema y la economía, carecen de inserción en el mercado y, por lo tanto, no tienen valor monetario. La valoración económica de los servicios de ecosistemas suministra información relacionada con los beneficios sociales resultantes del mejoramiento, protección y preservación del capital natural, permite cuantificar los costos de conservación, protección y mantenimiento de los recursos naturales, evalúa el monto de las obras de compensación que deben ir conexas a los proyectos de desarrollo, mejora la planificación y el aprovechamiento de los recursos naturales, contribuye con el diseño de las políticas de gestión ambiental, estima las implicaciones económicas provenientes de la contaminación, incentiva el uso racional de los recursos naturales por parte de la población y, por último, estima la productividad de un ecosistema (Randall 1985). El catastro ambiental también debe registrar la ubicación y descripción de las áreas de preservación, lo cual lo conecta fuertemente con los catastros físico y económico del modelo básico. Estos espacios con 
restricciones ambientales urbanas son los más depreciados por el mercado inmobiliario dado que son impropios para el desarrollo. Son espacios en los que el poder público tiene la intención de preservar pero que, por falta de fiscalización, rápidamente ingresan al mercado informal, cuya dinámica pasa a poseerlo, a generar lotes y a venderlos de manera diferenciada. Son espacios ocupados por asentamientos informales y por loteos pirata que no se identifican en el mapa catastral, sino hasta que se produce su desocupación o inclusive su regularización, aunque en este caso implicaría la desafectación de la restricción. Adelantarse a identificarlos es poner en práctica un proceso preventivo que se complementa con fiscalizaciones ostensivas para evitar las invasiones. Aun dentro del aspecto estructural, cabe mencionar el catastro de la red vial que puede ser estructurado para las redes de transporte terrestre y marítimo, la red hidrográfica e inclusive aeronáutica. Los ministerios, las secretarías, las direcciones nacionales y las jurisdicciones regionales y locales, en general, cuentan con cartografía de estas redes y las caracterizaciones de cada uno de sus elementos en bancos de datos asociados a través de un Sistema de información Geográfica (SIG). Estas redes viabilizan análisis espaciales de conectividad de las ciudades en forma global y, dentro del ámbito urbano, estas bases de datos se relacionan con el denominado catastro de calles. El trabajo coordinado de ambos permite determinar la accesibilidad de cada predio. El catastro de calles normalmente se estructura sobre la misma base cartográfica del catastro básico y en él constan los nombres de las calles, las numeraciones de los predios, el tipo de cobertura; así como informaciones útiles para planificar el tránsito y la distribución de señales, la recolección de basura, entre otros. Los datos de estos catastros influencian a otro grupo del aspecto estructural que corresponde al catastro de redes de servicios. Tal como el nombre lo indica, en sus bancos de datos constan las características y localización de los tubos, caños, cables, aéreos o subterráneos, así como los elementos que los sostienen como postes, torres, antenas, plataformas, conectores, grifos, válvulas, entre otros. Forman parte de este grupo los catastros de las redes de agua, energía eléctrica, alcantarillado, telefonía, gas, y cualquier otro servicio disponible en red en la ciudad. En muchas jurisdicciones latinoamericanas las empresas prestadoras de servicios fueron privatizadas, pero independientemente de su situación jurídica, actualmente es muy común ver que los catastros de la propia red y de los usuarios son administrados a través de sistemas de 
información que tienen un gran nivel de sofisticación. Los datos que constan en los SIG administrados por las compañías de servicios son muy relevantes para la determinación del valor de las parcelas, razón por la cual su conexión con el catastro municipal es fundamental. En aquellos casos en los que se produce intercambio de datos entre las instituciones, la transferencia es principalmente de datos alfanuméricos mientras que las bases cartográficas, aun cuando son digitales, presentan dificultades para su integración, puesto que normalmente están dibujadas en escalas o con precisiones diferentes y bajo sistemas de proyección cartográfica incompatibles. Esto dificulta la correlación interinstitucional debido a que las necesidades de la administración del catastro municipal, de las empresas de aguas y de energía eléctrica no son iguales en términos de detalle y precisión, siendo necesario articular discusiones para llegar a un nivel útil para todos. Una forma de articular a las instituciones que administran los catastros temáticos es a través de sistemas de información sobre todo a partir de la popularización de los SIG y la difusión de aplicativos abiertos y gratuitos. La estructuración de sistemas SIG con aplicativos de uso libre crea cultura de sistematización y tratamiento de la información geográfica para que, posteriormente, en los casos en que se evalúe como necesario, se implementen sistemas comerciales con mayor capacidad de análisis espacial.

Los aspectos geotecnológicos del catastro se van incorporando con diferentes velocidades a través de diversas herramientas. En los catastros dependientes de secretarías o direcciones de planeamiento es más frecuente encontrar plataformas para el desarrollo de estudios específicos. No obstante, en aquellos que dependen de secretarías de finanzas, hacienda o economía, la prioridad es la administración de datos alfanuméricos con fines tributarios, perdiendo gran parte del potencial de análisis espacial que los datos catastrales tienen. Estructurar un SIG coloca a un catastro municipal en la 5a. ola, pero no aún en la 6a. (ver figura 3).

Los instrumentos que tornan viable la integración horizontal y vertical de datos territoriales que conforman los aspectos institucionales del CTM son las IDE y los observatorios. Estos dos sistemas hacen interoperar a los catastros temáticos estructurados y mantenidos por diferentes instituciones, para lo cual es necesario establecer alianzas estratégicas, mecanismos formales de aproximación materializados en acuerdos de cooperación, convenios o 
consorcios a través de los cuales se comparten datos, información, personal, equipos, métodos de trabajo y todo lo que la creatividad de los administradores proponga.

Una IDE es un sistema integrado por un conjunto de recursos muy heterogéneos tales como datos, software, hardware, metadatos, servicios, estándares, personal, organización, marco legal, acuerdos, políticas, usuarios, entre otros, gestionado por una comunidad de actores para compartir información geográfica en la web de la manera más eficaz posible (Béjar y otros 2009).

La IDE no reemplaza a los SIG de cada una de las instituciones, sino que los relaciona para generar información más completa, actualizada y con gran nivel de detalle de la ciudad. Al definir estándares, la IDE permite que los sistemas operen entre ellos mientras cada aliado continúa trabajando su temática, con sus sistemas, de forma independiente y en paralelo.

Por definición, un CTM y una IDE tienen una gran vinculación. Una IDE es, en definitiva, el sistema que materializa un CTM, pues permite visualizar y poner en práctica los conceptos de multifinalidad. Mientras las IDE propician la interoperabilidad tecnológica, el CTM propicia la interoperabilidad institucional.

En Ecuador el COOTAD en su art. 139 atribuye a los gobiernos locales potestades de formación y administración de los catastros inmobiliarios urbanos y rurales. Esta disposición se complementa con el art. 147, el cual afirma que el gobierno central, a través del ministerio responsable, es decir, el MIDUVI, dictará las políticas nacionales para garantizar el acceso universal a este derecho y mantendrá, en coordinación con los gobiernos de administración descentralizada (GAD), un catastro territorial que mantenga coherencia a nivel nacional.

En Ecuador hay numerosas iniciativas de estructuración de IDE entre las cuales se puede mencionar el Sistema Nacional de Información de la Secretaria Nacional de Planificación y Desarrollo 〈http://sni.gob.ec/〉 y el portal impulsado por el Instituto Geográfico Militar 〈http://www.geoportaligm. gob.ec/portal/ $>$. Una encuesta aplicada por el MIDUVI a inicios de 2014 en Ecuador, dirigida a los responsables de la gestión catastral en 132 GAD municipales, mostró que el $25 \%$ de municipios no dispone de un sistema de información geográfica, y que, si bien el 92\% cuenta con internet de banda ancha, el 66\% considera que las Direcciones de Catastro no disponen de 
la infraestructura tecnológica necesaria. Esta encuesta muestra también que hay mucho espacio para la integración del catastro municipal con órganos del gobierno local y externos.

\section{Un sistema nacional de catastro para Ecuador}

Las estructuras y las herramientas que caracterizan a los catastros territoriales del Ecuador no atienden a todas las necesidades de los planificadores, fiscalistas y economistas. Para revertir esta situación, el MIDUVI, como ente rector, puso en marcha junto a los principales actores del quehacer catastral del país en la estructuración del sistema nacional de catastro (SNC). El ministerio busca establecer un sistema nacional de catastro, y no un sistema de catastro nacional. El SNC tiene como objetivo establecer lineamientos generales que orienten a los administradores y permitan al país contar con continuidad cartográfica y de valores de los inmuebles, respetando las potestades de los GAD en el área.

El SNC se apoya en dos pilares fundamentales: las Normas de Avalúos y de Catastro, las cuales pasarán a regular todas las actividades catastrales en el país (figura 4). Estas normas están siendo construidas por comités con-

Figura 4

\section{Estructura del Sistema Nacional de Catastro propuesto para Ecuador}

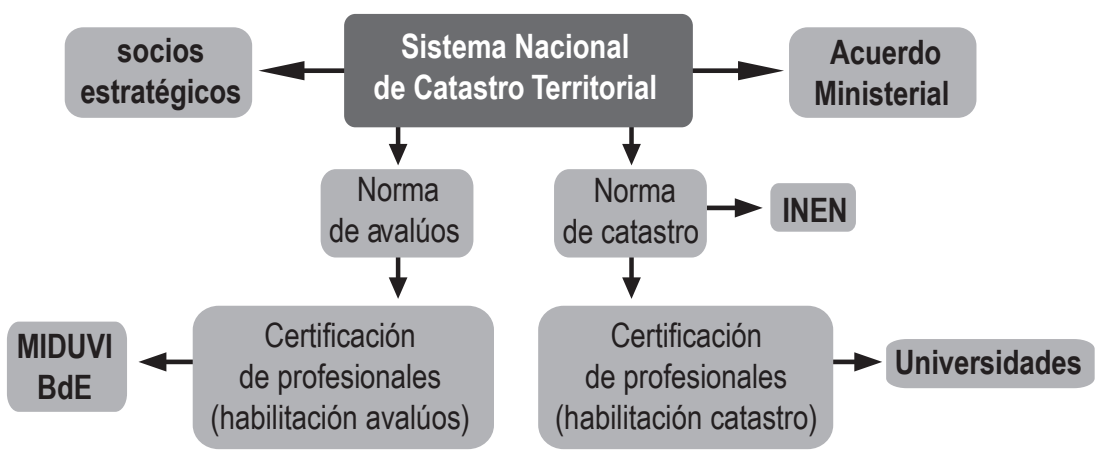

Fuente: el autor.

Elaboración propia. 
formados por técnicos representantes de diversas instituciones públicas y privadas, nacionales e internacionales, GAD y la academia, con supervisión del Instituto Ecuatoriano de Normalización (INEN). El SNC ya comenzó a instrumentalizarse a través del Acuerdo Ministerial No. 022/2014 del MIDUVI, el cual establece los lineamientos para el mantenimiento catastral. De forma paralela con la redacción de las normas, se está trabajando en la estructuración de los cursos de formación y exámenes de certificación de profesionales que los habiliten para el ejercicio de actividades en las áreas afines del SNC.

La estructuración del SNC se está dando en un contexto de creciente madurez conceptual y voluntad política. Manteniendo las potestades municipales, cada administración desarrollará su forma de implementarlo, incorporando aliados y sumando aspectos del catastro territorial de forma paulatina y modular, siempre dentro de los parámetros establecidos por el MIDUVI.

\section{El catastro municipal}

El catastro municipal debe estar preparado para dar respuestas a intervenciones de cualquier escala: las que son solventadas con fondos públicos o capitales privados; las que afectan a un único predio o contemplan varios; las que causan impactos mínimos en el medioambiente hasta las que exijan acciones que compensen las alteraciones provocadas. Sus funciones son, por un lado, tornar disponibles los datos que se le requieran, y, por otro, capturar los datos y actualizar sus registros alfanuméricos y cartográficos en función de las modificaciones que cada intervención haya introducido en la ciudad.

El catastro, cuando es estructurado bajo el modelo multifinalitario, aporta los datos necesarios a los procesos asociados al planeamiento, y se nutre de datos derivados de su aplicación. Entre las aplicaciones del CTM en la definición de políticas públicas se destacan sus relaciones con los proyectos de (re)desarrollo urbano a gran escala, su contribución con la disminución de la informalidad urbana y sus interacciones con el financiamiento urbano a través de la recuperación de plusvalías y la tributación inmobiliaria. 


\section{EI CTM y el planeamiento urbano}

La frase que afirma: "el catastro debe estar al servicio del planeamiento" no es nueva pero, a pesar de todo lo que se ha escrito y dicho sobre los deberes y derechos del catastro, aún es posible hacer análisis más detallados. El catastro municipal participa en prácticamente todas las intervenciones urbanas que derivan de acciones administrativas del Estado o de emprendimientos de un ciudadano o de un pequeño grupo que se producen en las ciudades y su nivel de involucramiento depende de la escala de cada uno de ellos. La expansión, horizontal y vertical, de las ciudades latinoamericanas está condicionada por una serie de cambios demográficos y económicos en la región. Desde grandes proyectos para sectores sociales de ingresos medios y bajos, hasta las exclusivas gated communities (urbanizaciones enrejadas) para los grupos de altos ingresos. En paralelo, los asentamientos informales continúan con falta de equipamientos y de servicios urbanos como transporte público, vías de acceso adecuadas, suministro de agua y alcantarillado. La tendencia de expansión hacia esas áreas periféricas sobrevaluadas, pero al mismo tiempo carentes de servicios, contrasta con la reducción de la actividad residencial en áreas centrales provistas de equipamientos y servicios básicos. Conforme estas zonas urbanas de suelo subutilizado y vacante se vuelven menos pobladas y más devaluadas, el ciclo del deterioro va profundizándose (Lungo 2001).

En el marco del proyecto de Ley Orgánica de Ordenamiento Territorial, Uso y Gestión del Suelo del MIDUVI, la urbanización es entendida como la dotación de infraestructuras y servicios a los asentamientos concentrados de población. La norma prevé distintas formas de gestión que responden a las variadas necesidades de transformación de suelo que puedan darse. Los municipios que necesiten expandir el suelo urbano, o regenerarlo, podrán delimitar unidades de actuación urbanística, que son ámbitos no solo para la ejecución de las obras, sino también para la reestructuración de la propiedad y la distribución equitativa de los beneficios y cargas derivados de las intervenciones. Así, son los gobiernos autónomos descentralizados municipales o metropolitanos quienes deciden dónde se hace ciudad, con qué usos y calidad, a través de los planes de uso y gestión del suelo y sus instrumentos urbanísticos complementarios, aprobados con participación 
ciudadana. ¿Cómo hacer esto sin un catastro estructurado bajo el modelo multifinalitario?

La normativa urbanística, cuando existe, tiende a ser tan rigurosa que solo una parte de la ciudad tiene la capacidad de respetarla, mientras que la otra desarrolla viviendas y barrios sin observarla. Como consecuencia, una parte de la ciudad es producida de forma regular y la otra parte de forma irregular. Estas dualidades implican la existencia de un mercado de suelo formal, regulado y controlado por un marco de derecho privado y las normas urbanísticas; y un mercado informal que se desarrolla con reglas propias, aunque articuladas con las del mercado formal. La exclusión urbana, derivada de la dinámica del mercado orientada por un derecho privatista, acaba manifestándose en el catastro físico, cuyos mapas muy frecuentemente registran solamente a las parcelas sometidas al régimen de propiedad privada, desconsiderando a los sectores informales de la ciudad. Los asentamientos están en la foto pero no están en los mapas. Los conceptos y afirmaciones vertidos en los párrafos anteriores (Alfonsin 2013) permiten identificar algunas relaciones entre los procesos de desarrollo urbano y sus impactos en los diferentes aspectos del catastro territorial.

En la encuesta aplicada por el MIDUVI en 2014, casi el 60\% de los gobiernos locales reconocen tener asentamientos informales en el área urbana y casi el 70\% en el área rural de su jurisdicción. Aun así, para la mayoría de los catastros, la ciudad es un gran espacio de propiedad privada (materializada en terrenos baldíos, casas, apartamentos, condominios y otros) con alternancia de propiedad pública (calles, plazas, edificios de la administración gubernamental, entre otros). El aislamiento institucional y la parcialidad del modelo catastral básico impiden comprender y mapear la dinámica urbana y hace perder oportunidades.

\section{EI CTM y el financiamiento urbano}

Al hablar de financiamiento urbano en América Latina lo primero en que se piensa es en el impuesto predial. Su relación con el catastro territorial es obvia, de hecho, muchos consideran que la razón de la existencia de un catastro es dar soporte a ese impuesto. No obstante, es fundamental que pro- 
ductores y usuarios de datos catastrales comprendan que el financiamiento de las ciudades va más allá, se hace a través de los tributos, término genérico que comprende impuestos, tasas y contribuciones.

La definición de la política tributaria puede seguir diferentes criterios, no obstante, independientemente del que se elija, hay seis principios que, según De Cesare (2012), deben ser seguidos: equidad, capacidad de pago, universalidad, legalidad, administración eficiente y transparencia; cada uno de los cuales pone en evidencia la importancia que un catastro territorial tiene para alcanzarlos.

En su art. 57 el COOTAD atribuye al Concejo Municipal la regulación, mediante ordenanza, de la aplicación de tributos previstos en la ley; crear, modificar, exonerar o extinguir tasas y contribuciones especiales por los servicios que presta y obras que ejecute. En este sentido, contar con un mapa de valores referido al mercado inmobiliario es esencial, y respetar esos valores es el único camino para alcanzar la equidad tributaria. En Ecuador una práctica frecuente ha sido alterar valores de los inmuebles de acuerdo a ciertos lineamientos para definir la política tributaria. Esta forma de actuar desestimula a los técnicos de los catastros, pues su trabajo es desconsiderado; provoca inequidades y reduce los ingresos municipales, todo lo cual deriva en ciudades con menos infraestructura, equipamiento y servicios. Los valores de los inmuebles no son políticos, pueden y deben ser determinados de forma científica, las tarifas deben ser definidas políticamente, y es esa la razón por la cual el COOTAD establece rangos. Para revertir la situación el país necesita un catastro territorial que torne disponible datos económicos y métodos de valoración referenciados al mercado inmobiliario como única alternativa para mejorar la precisión y la uniformidad, datos físicos correspondiente a la ubicación, forma y dimensiones de los predios y sus construcciones, datos jurídicos, informaciones sobre las redes de servicios que sirven a los predios, restricciones ambientales y urbanísticas que eventualmente los afectan, bien como datos sociales sobre capacidad contributiva de los propietarios o poseedores. Mientras las reestructuraciones de los catastros hacia el modelo multifinalitario se van construyendo, la actualización de las bases de datos existentes ayuda a mejorar el desempeño de la recaudación.

El art. 497 del COOTAD trata del impuesto predial y su actualización, pero aún en las jurisdicciones que cuentan con catastros territoriales razona- 
blemente bien organizados bajo el modelo básico, las recaudaciones son muy bajas en relación al potencial.

Otro tributo muy importante en el Ecuador es la contribución especial de mejoras (CEM), la cual es motivada por un beneficio real o presuntivo proporcionado a las propiedades inmuebles urbanas por la construcción de cualquier obra pública. Se aplica solo para obras ya realizadas y se concentra mayormente en la construcción y repavimentación de vías, aunque puede ser sobre redes de agua potable, alcantarillado, parques y otras obras que los municipios determinen. La CEM recupera costos directos, estudios, indemnización y costos financieros con un bajo nivel de complejidad y supone que los beneficios de la infraestructura se capitalizan en el valor del predio. El art. 264 de la Constitución de la República de Ecuador estipula que los gobiernos municipales tienen la competencia, entre otras, de modificar o suprimir tasas y contribuciones especiales de mejoras. El catastro cubre apenas $60 \%$ de los lotes urbanos, y el precio del suelo registrado, que tiene únicamente una finalidad impositiva, se encuentra distante de los practicados en el mercado a pesar de la obligatoriedad de la actualización bienal. En este sentido, la CEM apareció como un instrumento válido para efectuar una recuperación de los costos de los proyectos, a la vez que permite recuperar, cuando menos de forma parcial, la plusvalía generada por la obra pública y el desarrollo urbano.

\section{Perspectivas catastrales para Ecuador}

La diversidad de modernas tecnologías y conceptos acerca de los sistemas catastrales y su administración, que están surgiendo en los últimos años, abren nuevas perspectivas para los gobiernos locales. A corto plazo la incorporación de técnicas de valuación basadas en modelos econométricos y geoestadística referidos al mercado, la estructuración de SIG libres en las jurisdicciones que no cuentan todavía con sistemas y el uso de productos cartográficos no convencionales son tendencias que se consolidarán. A mediano plazo, el modelo multifinalitario se difundirá en muchas jurisdicciones puesto que las leyes catastrales ya lo contemplan. En este período también se espera que los registros gráficos catastrales se reestructuren a representaciones 3D usando los datos en ambiente SIG, pasando a representar no solamente la ciudad formal sino también la informal. Para todo esto serán necesarios más 
profesionales del catastro, por lo cual más cursos de grado y posgrado serán imperiosos. Finalmente, a largo plazo, la perspectiva es que los más modernos conceptos de catastro 3D y crowdsourcing (externalización de ideas) serán adaptados a la realidad latinoamericana. De forma similar, el Land Administration Domain Model (LADM), ocupará espacios en los proyectos de reestructuración de los catastros, obteniéndose resultados que inspiren la consolidación de sistemas de información territorial espaciales y cambien la visión de la legislación urbanística y ambiental. Los catastros territoriales estructurados en Ecuador que contemplan por lo menos datos económicos, físicos y jurídicos mantendrán en los próximos años esa estructura básica; no obstante, incorporarán sistemas y métodos de actualización más eficientes y eficaces que los tradicionalmente utilizados.

En el área económica, la tendencia fuerte es consolidar observatorios de valores liderados por las instituciones catastrales. Los excelentes resultados arrojados por los observatorios existentes en diferentes ciudades latinoamericanas son suficientemente elocuentes y pedagógicos, como para mostrar a los administradores que es ventajoso contar con una estructura de monitoreo del mercado inmobiliario que se articule a los actores más involucrados con la compraventa de inmuebles, créditos hipotecarios, traslación de dominio, entre otros. En tal sentido, la elaboración de mapas de valor basados en técnicas econométricas con apoyo de geoestadística, actualmente de conocimiento casi exclusivo de consultores particulares, se incorporarán paulatinamente en los equipos de funcionarios públicos de los catastros territoriales.

En el área cartográfica los levantamientos realizados a través de métodos topográficos y fotogramétricos darán espacio a los mapeos realizados con productos obtenidos por vehículos aéreos no transportados (VANT y LIDAR). Aun dentro del área tecnológica, se invertirá la lógica de implementación de SIG. Jurisdicciones con escasos recursos o de pequeñas dimensiones comenzarán a incorporar software libre en vez de quedarse esperando financiamiento para adquirir aplicativos comerciales, los cuales serán prioritarios después de crear la cultura catastral digital. En términos estructurales el modelo básico se moverá hacia el multifinalitario, con lo cual la administración de la institución responsable por el catastro se desplazará de los organismos de recaudación hacia los de planeamiento. De esta manera los catastros territoriales continuarán teniendo un rol fundamental en la definición de la polí- 
tica tributaria, incrementando su protagonismo en la definición de políticas urbanas, ofreciendo más datos a los planificadores al suministrar informaciones sistemáticas provenientes de múltiples fuentes, y a los economistas urbanos, contribuyendo de forma efectiva con la implementación de los más modernos instrumentos de financiamiento.

La diversidad de modernas tecnologías y conceptos acerca de los sistemas catastrales y su administración, que están surgiendo en los últimos años, abren nuevas perspectivas para los gobiernos locales. A corto plazo la incorporación de técnicas de valuación basadas en modelos econométricos y geoestadística referidos al mercado, la estructuración de SIG libres en las jurisdicciones que no cuentan todavía con sistemas y el uso de productos cartográficos no convencionales son tendencias que se consolidarán.

A mediano plazo el modelo multifinalitario se difundirá en muchas jurisdicciones puesto que su estructura está en el imaginario de los administradores hace años. Su implementación definitivamente cambiará la gestión municipal.

\section{Referencias}

Alfonsin, Betania. 2013. "Una mirada dialéctica sobre la informalidad urbana". En Diego Alfonso Erba, edit., Definición de políticas de suelo urbano en América Latina: teoría y práctica. 〈https://www.lincolninst.edu/publications/books/definicion-politicas-suelo-ur bano-en-america-latina.

Aulestia, Diego, y Vanessa Rodríguez. 2014. "Incentivos por el cobro de contribución especial de mejoras para el financiamiento de la infraestructura pública". En Martin Smolka y Fernanda Furtado. Instrumentos notables de politicas de suelo en América Latina. Cambridge: Lincoln Institute of Land Policy. 〈http://www.lincolninst.edu/pubs/2414_Instru mentos-notables-de-pol\%C3\%ADticas-de-suelo-en-Am\%C3\%A9rica-Latina〉.

Béjar, Rubén, Miguel Ángel Latre, Javier Nogueras-Iso, Pedro Muro-Medrano y Javier Zarazaga-Soria. 2009. "Systems of Systems as a Conceptual Framework for Spatial Data Infrastructures". International Journal of Spatial Data Infraestuctures Research, No. 4: 201-217.

Código Orgánico de Organización Territorial, Autonomía y Descentralización (COOTAD). ROS, No. 303 (19 de octubre de 2016), art. 502.

Constitución de la República del Ecuador [2008]. [Quito].

De Cesare, Claudia. 2012. "Improving the Performance of the Property Tax in Latin Ame- 
rica”. Policy Focus Report. (Cambridge: Lincoln Institute of Land Policy). 〈http://www. lincolninst.edu/publications/policy-focus-reports/improving-performance-property-tax-la tin-america).

Ecuador. Acuerdo Ministerial No. 22, 12 de julio de 2013.

Erba, Diego. 2007. "Catastro multifinalitario aplicado a la definición de políticas de suelo urbano". Cambridge: Lincoln Institute of Land Policy. 〈http://www.lincolninst.edu/pubs/1306 Catastro-multifinalitario-aplicado-a-la-definici\%C3\%B3n-de-pol\%C3\%ADticas-de-sue lo-Urbano).

--- 2008. "El catastro territorial en América Latina y el Caribe". Cambridge: Lincoln Institute of Land Policy. «ttps://www.lincolninst.edu/pubs/1542_Catastro-e-in formaci\%C3\%B3nterritorial-en-Am\%C3\%A9rica-Latina).

Francia. 1804. Código Napoleónico, 21 de marzo de 1804.

Gómez-Baggethun, Erick, y Rudolf de Groot. 2007. "Capital natural y funciones de los ecosistemas: explorando las bases ecológicas de la economía”. Ecosistemas 16, No. 3: 4-14.

Ley Orgánica de Ordenamiento Territorial Uso y Gestión del Suelo. ROS, No. 790, 5 de julio de 2016.

Lungo, Mario. 2001. "Expansión urbana y regulación del uso del suelo en América Latina". En Martim Smolka y Laura Mullahy, edit., Perspectivas urbanas 2007: temas críticos en políticas de suelo en América Latina. Cambridge: Lincoln Institute of Land Policy. 〈https:// www.lincolninst.edu/sites/default/files/pubfiles/perspectivas-urbanas-cd-full.pdf〉.

Randall, Allan. 1985. Economía de los recursos naturales y política ambiental. México: Limusa. 